\title{
Absorption Spectra of Broadened Sodium Resonance Lines in Presence of Rare Gases
}

H.K. Chung, M. Shurgalin, J.F. Babb

This article was submitted to $16^{\text {th }}$ International Conference on Spectral Line Shapes (ICSLS), Berkeley, California, June 3-7, 2002

U.S. Department of Energy

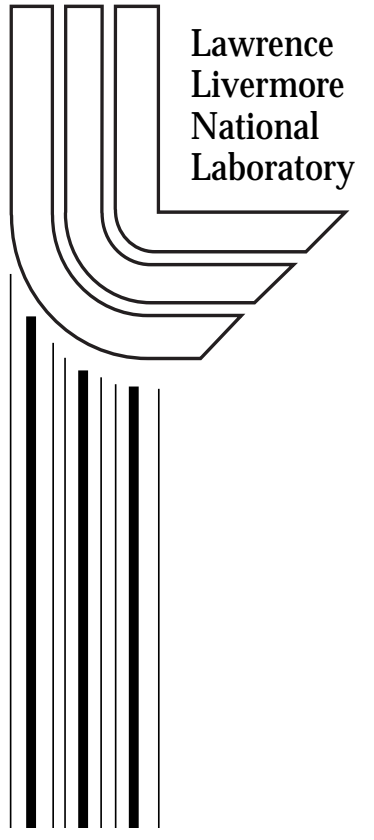

\section{September 11, 2002}




\section{DISCLAIMER}

This document was prepared as an account of work sponsored by an agency of the United States Government. Neither the United States Government nor the University of California nor any of their employees, makes any warranty, express or implied, or assumes any legal liability or responsibility for the accuracy, completeness, or usefulness of any information, apparatus, product, or process disclosed, or represents that its use would not infringe privately owned rights. Reference herein to any specific commercial product, process, or service by trade name, trademark, manufacturer, or otherwise, does not necessarily constitute or imply its endorsement, recommendation, or favoring by the United States Government or the University of California. The views and opinions of authors expressed herein do not necessarily state or reflect those of the United States Government or the University of California, and shall not be used for advertising or product endorsement purposes.

This is a preprint of a paper intended for publication in a journal or proceedings. Since changes may be made before publication, this preprint is made available with the understanding that it will not be cited or reproduced without the permission of the author. 


\title{
Absorption spectra of broadened sodium resonance lines in presence of rare gases
}

\author{
H.-K. Chung* ${ }^{*}$ M. Shurgalin ${ }^{\dagger}$ and J. F. Babb ${ }^{* *}$ \\ ${ }^{*}$ Lawrence Livermore National Laboratory, Livermore CA 94550 \\ ${ }^{\dagger}$ OmniGuide Communications Inc., Cambridge, MA 02139 \\ ** ITAMP, Harvard-Smithsonian Center for Astrophysics, Cambridge, MA 02138
}

\begin{abstract}
The pressure broadening of alkali-metal lines is a fundamental problem with numerous applications. For example, the sodium resonance lines broadened by xenon are important in the production of broad spectra emitted in the HPS (High-Pressure Sodium) lamp and they potentially can be used for gas condition diagnostics. Broadened absorption lines of alkali-metal atoms are prominent in the optical spectra of brown dwarfs and understanding the broadening mechanism will help elucidate the chemical composition and atmospheric properties of those stars.

The far-line wing spectra of sodium resonance lines broadened by rare gases are found to exhibit molecular characteristics such as satellites and hence the total absorption coefficients for vapors of $\mathrm{Na}$ atoms and perturbing rare gas atoms can be modeled as Na-RG (rare gas) molecular absorption spectra. In this work, using carefully chosen interatomic potentials for Na-RG molecules we carry out quantum-mechanical calculations for reduced absorption coefficients for vapors composed of $\mathrm{Na}-\mathrm{He}, \mathrm{Na}-\mathrm{Ar}$, and $\mathrm{Na}-\mathrm{Xe}$. Calculated spectra are compared to available experimental results and the agreement is good in the measured satellite positions and shapes.
\end{abstract}

\section{INTRODUCTION}

Line broadening of sodium resonance lines in presence of perturbing atoms have been extensively studied over several decades. Due to interatomic interaction between $\mathrm{Na}$ atom and perturbers, far line wings of the broadened lines exhibit characteristics of molecular spectra. Therefore, experimental methods such as laser-induced fluorescence, emission and absorption spectroscopy have been used to study properties of interatomic interactions such as interaction potentials. In addition to the fundamental studies of molecular physics, thermal emission and absorption spectra of sodium resonance lines are studied in the context of various applications such as the modeling of high-pressure sodium lamps and optical diagnostics of combustion and chemical reactions.

Theoretically, the far line wing spectra has been studied with classical and semiclassical methods. These methods give a good description of gross features of absorption and emission spectra. However, recent high-precision measurements of absorption coefficients [1] reveal more detailed features such as ro-vibration lines and satellite structures in the far line wings of sodium resonance lines when broadened by the same or different perturbing atoms requiring more precise theoretical descriptions. Accurate quantummechanical methods in molecular spectroscopy are not usually applied to the spectral calculations for high temperature gas partly because the uncertainty of available molecular potentials is comparable to that of the classical and semi-classical methods and partly 
TABLE 1. Molecular potentials used in the calculations

\begin{tabular}{cc}
\hline Rare gas & $\mathbf{X}^{2} \Sigma^{+}, \mathbf{A}^{2} \Pi$ and $\mathbf{B}^{2} \Sigma^{+}$potentials \\
\hline $\mathrm{He}$ & Theodorakopoulosis and Petsalakis [8] \\
$\mathrm{Ar}$ & Kerner et al $[9]$ \\
$\mathrm{Xe}$ & Baumann et al. $\left(\mathrm{X}^{2} \Sigma^{+}\right.$and $\left.\mathrm{A}^{2} \Pi\right)[10]$ Düren-type potential function $\left(\mathrm{B}^{2} \Sigma^{+}\right)[11,7]$ \\
\hline
\end{tabular}

because of the computational limitations resulting from the difficulty of accounting for all the partial waves contributing to the total spectra. Recently, however, accuracies for atomic and molecular data have been greatly improved by utilizing various methods such as laser spectroscopy, photoassociation spectroscopy, Feshbach resonance, and ultracold collisions, and also recent advances in computer speed and techniques of parallel and distributed processing made it possible to consider the convergent number of partial waves involved in calculations. In this work, using the PVM (Parallel Virtual Machine) technique, we carry out quantum-mechanical calculations of absorption coefficients for far line wing spectra of sodium resonance lines broadened by rare gases.

\section{QUANTUM-MECHANICAL METHOD}

When broadened by rare gases, absorption coefficients of sodium resonance lines arising from $3 \mathrm{~s}$ to $3 \mathrm{p}$ transitions can be modeled as molecular spectra of transitions within dimolecules composed of sodium and rare gas atoms. Absorption transitions occur from the lower molecular states which approach the absorbing sodium $(3 s)$ and the perturbing rare gas atomic states at a long-range limit to the upper molecular states which approach the excited sodium ( $3 p)$ and the spectator rare gas atomic states. Depending on whether the molecular potentials have bound states formed by sodium and rare gas atoms, the total spectra consist of four possible transitions; bound-bound, bound-free, free-bound, free-free transitions. Details of quantum-mechanical formulation of each transition are described in our earlier papers [2, 3].

Most important are the molecular potentials involved in the radiative transitions and a lot of care was taken into using the most accurate molecular potentials of sodium-rare gas di-molecules. Molecular potentials are roughly divided into short-range potentials which can be obtained by ab initio calculations or experimental methods such as laser spectroscopy or line broadening measurements and long-range potentials normally described as $V(R)=-C_{6} / R^{6}$ where van der Waals forces are dominant. We investigated the potentials available in the literature by calculating theoretical values of thermal gas parameters such as diffusion coefficients and index of refraction of sodium matter waves using those potentials and comparing with the measured values [4, 5]. Measured satellite positions $[1,6,7]$ were applied to select and modify molecular potentials as well. Molecular potentials of our choice are listed in Table. 1-2 for $\mathrm{Na}-\mathrm{He}$ and $\mathrm{Na}-\mathrm{Ar}$ and NaXe molecules. Details of our studies on molecular potentials will be published elsewhere shortly [7]. 
TABLE 2. $C_{6}$ values in atomic units for long-range molecular potentials $V(R)=-C_{6} / R^{6}$ used in calculations

\begin{tabular}{cccc}
\hline Rare gas & $\mathbf{X}^{2} \Sigma^{+}$potential & $\mathbf{A}^{2} \Pi$ potential & $\mathbf{B}^{2} \Sigma^{+}$potential \\
\hline $\mathrm{He}$ & $26.2[12]$ & $49.2[13]$ & $71.4[13]$ \\
$\mathrm{Ar}$ & $190.0[12]$ & $534.7[9]$ & $717.8[9]$ \\
$\mathrm{Xe}$ & $455.9[12]$ & $897.4[14]$ & $1095.4[14]$ \\
\hline
\end{tabular}

\section{COMPARISONS WITH MEASUREMENTS}

Experimentally, the absorption coefficients of sodium vapor at $900\left(^{+} 10^{-} 50\right) \mathrm{K}$ in presence of various rare gases were measured in the range from $400 \mathrm{~nm}$ to $850 \mathrm{~nm}$. With the sodium atomic densities obtained accurately using the anomalous dispersion method [1], density-independent reduced absorption coefficients of $\mathrm{Na}-\mathrm{Na}$ and $\mathrm{Na}$-Rare gases were obtained simultaneously. The measured reduced absorption coefficients (upper curves) for $\mathrm{Na}-\mathrm{He}, \mathrm{Na}-\mathrm{Ar}$ and $\mathrm{Na}-\mathrm{Xe}$ are compared with the calculated values (lower curves) in Fig. 1. The blue wing absorption arises from transitions between the $\mathrm{X}^{2} \Sigma^{+}$molecular state to the $\mathrm{B}^{2} \Sigma^{+}$molecular state and the red wing from transitions between the $\mathrm{X}^{2} \Sigma^{+}$ molecular state to the $\mathrm{A}^{2} \Pi$ molecular state. Since the ground electronic states of $\mathrm{Na}$ and rare gas di-molecules are weakly bound, most absorption occurs from free-bound and free-free transitions and the absorption spectra should be smooth without ro-vibrational structures ${ }^{1}$.

In the blue wings notable features are the primary peaks occurring at 530, 555 and $560 \mathrm{~nm}$ for $\mathrm{Na}-\mathrm{He}, \mathrm{Na}-\mathrm{Ar}$ and $\mathrm{Na}-\mathrm{Xe}$ respectively. Quantum-mechanical calculations reproduce the primary peaks at the measured positions. In addition to the primary peaks, there are weaker secondary peaks shown for $\mathrm{Na}-\mathrm{Ar}$ and $\mathrm{Na}-\mathrm{Xe}$ and they are also reproduced in calculations. The semi-classical method suggested by Bieniek and Streeter $[15,16]$ also yields results similar to the quantum-mechanical method, however, other classical and semi-classical methods [17] fail to describe the primary and the secondary peaks altogether. While our results give a overall good agreement, we found that the absolute values of calculated intensities are slightly lower than the measured values and the sources of discrepancies are not known yet. We also found that the secondary peaks require more stringent tests of molecular potentials than primary peaks and the shapes and the positions of the secondary peaks can be applied to refine molecular potentials.

\footnotetext{
1 The structures in the experimental red wing spectra are believed to be merely the remnants of sodium dimer spectra appearing because reduced absorption coefficients are extracted from spectra of total absorption coefficients for the gas mixtures.
} 

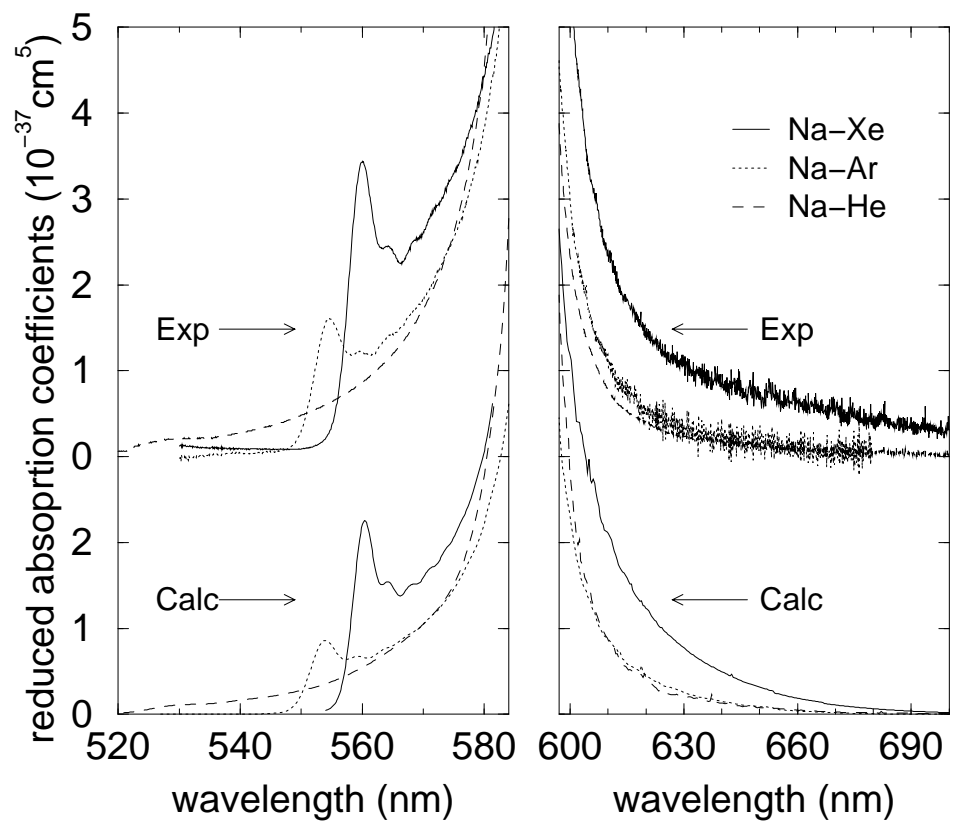

FIGURE 1. Comparisons for Na-rare gas molecules of reduced absorption coefficients between experiment at temperature of $900+10-50 \mathrm{~K}$ (upper curves) and theory at temperature of $900 \mathrm{~K}$ (lower curves).

\section{APPLICATIONS}

Pressure broadening of atomic resonance lines is a key ingredient of various lighting sources. In particular, our research is germane to the spectra of high-pressure sodium lamps [18]. Our measured and calculated absorption coefficients might be utilized for improved emission models.

Another intriguing application of the present research is found in astrophysics. The spectra of brown dwarfs contains alkali-metal atom resonance lines pressure-broadened predominantly by the perturbing gases $\mathrm{He}$ and molecular hydrogen [19]. The resonance lines of $\mathrm{K}$ and $\mathrm{Na}$ are particularly prominent and improved modeling of their broadening may lead to better knowledge of atmospheric composition, effective temperature and gravity $[20,21]$.

\section{SUMMARY}

High precision measurements were used to obtain the absorption spectra of sodium resonance lines in presence of rare gases. The absorption coefficients show interesting peak structures in the blue wings. We demonstrated that quantum-mechanical methods can be applied to describe the peak and the overall features of far wing spectra of sodium resonance lines when broadened by rare gases. Our results show that the structures provide a good test of molecular potentials of sodium-rare gas di-molecules. 


\section{ACKNOWLEDGMENTS}

This work is supported in part by the NSF, grant PHY-9724713 and a partial support for H.K.Chung is provided under the auspices of the U.S. Department of Energy by University of California Lawrence Livermore National Laboratory under contract No. W-7405-Eng-48.

\section{REFERENCES}

1. Shurgalin, M., Parkinson, W. H., Yoshino, K., Schoene, C., and Lapatovich, W. P., Meas. Sci. Technol., 11, 730-737 (2000).

2. Chung, H.-K., Kirby, K., and Babb, J. F., Phys. Rev. A, 60, 2002 (1999).

3. Chung, H.-K., Kirby, K., and Babb, J. F., Phys. Rev. A, 63, 032516 (2001).

4. Ager III, J. W., and Howard, C. J., J. Chem. Phys., 85, 3469 (1986).

5. Schmiedmayer, J., Chapman, M. S., Ekstron, C. R., Hammond, T. D., Whinger, S., and Pritchard, D. E., Phys. Rev. Lett., 74, 1043 (1995).

6. Ch'en, S. Y., and Jr., R. A. W., Physica, 27, 497 (1961).

7. Chung, H.-K., Shurgalin, M., and Babb, J. F., Experimental and theoretical studies of sodium resonance line broadening in presence of rare gases (2002), in progress.

8. Theodorakopoulos, G., and Petsalakis, I. D., J. Phys. B: At. Mol. Opt. Phys., 26, 4367 (1993).

9. Kerner, C., and Meyer, W., Ph. D. thesis, Universität Kaiserslautern, Kaiserslautern, Germany, 1995.

10. Baumann, P., Zimmermann, D., and Brühl, R., J. Mol. Spectros., 155, 277 (1992).

11. Düren, R., Hasselbrink, E., and Hillrichs, G., J. Chem. Phys., 89, 2822 (1988).

12. Derevianko, A. (2000), private communication.

13. Leo, P. J., Peach, G., and Whittingham, I. B., J. Phys. B: At. Mol. Opt. Phys., 33, 4779 (2000).

14. Mahan, G. D., J. Chem. Phys., 50, 2755 (1969).

15. Bieniek, R. J., and Streeter, T. J., Phys. Rev. A, 28, 3328 (1983).

16. Sato, Y., Nakamura, T., Okunishi, M., Ohmori, K., Chiba, H., and Ueda, K., Phys. Rev. A, 53, 867 (1996).

17. Szudy, J., and Baylis, W. E., J. Quant. Spectrosc. Radiat. Transfer, 15, 641 (1975).

18. de Groot, J. J., and van Vliet, J. A. J. M., The high pressure sodium lamp, Philips Technical Library, Kluwer Techn. Boeken, Deventer, 1986.

19. Burrows, A., Hubbard, W. B., Lunine, J. I., and Liebert, J., Rev. Mod. Phys., 73, 719 (2001).

20. Schweitzer, A., Gizis, J., Hauschildt, P., Allard, F., and Reid, I., Ap.J., 555, 368 (2001).

21. Burrows, A., Burgasser, A. J., Kirkpatrick, J. D., Liebert, J., Milsom, J. A., Sudarsky, D., and Hubeny, I. (2002), astro-ph/0109227 Ap.J. in press 2002. 